\title{
TIMELESS is a Potential Molecular Marker in Glioma: A Study Based on Multiple Databases and Methods
}

\section{Zhendong Liu}

Henan Provincial People's Hospital

\section{Zhishuai Ren}

Henan Provincial People's Hospital

\section{Zhibin Han}

First Affiliated Hospital of Harbin Medical University

\section{Xingbo Cheng}

First Affiliated Hospital of Harbin Medical University

\section{Wang Zhang}

First Affiliated Hospital of Harbin Medical University

\section{Binfeng Liu}

Henan Provincial People's Hospital

Jialin Wang

Henan Provincial People's Hospital

Yanzheng Gao ( $\nabla$ yanzhenggaohn@gs.zzu.edu.cn )

Henan Provincial People's Hospital https://orcid.org/0000-0003-3690-1954

\section{Research}

Keywords: glioma, TIMELESS, biomarker, oncology, small molecule compound.

Posted Date: December 23rd, 2020

DOI: https://doi.org/10.21203/rs.3.rs-132735/v1

License: (1) This work is licensed under a Creative Commons Attribution 4.0 International License. Read Full License 


\section{Abstract}

Background: In recent years, some studies have shown that TIMELESS, as an oncogene, is involved in the malignant progression of cancers. However, its relationship with the prognosis of glioma patients is rarely reported. Our purpose is to explore the role of TIMELESS in glioma.

Main body: Based on 1814 glioma samples from multiple databases such as TCGA, CGGA, GEO, we use a variety of bioinformatics methods to verify the mechanism of action of TIMELESS in glioma from mRNA to protein, from appearance to mechanism analysis, from clinical features to prognosis. Then, use the CMap tool to predict potential drugs that inhibit the expression of TIMELESS. First, we found TIMELESS is highly expressed in glioma from the mRNA level to the protein level. Second, TIMELESS is an independent risk factor for prognosis and has a suitable clinical diagnosis value in glioma. It was also positively correlated with WHO grade, age, histology, and negatively correlated with IDH1 mutation and 1p19q codeletion. Third, Base excision, cell cycle, and mismatch repair pathway were activated by TIMELESS in glioma. At last, we predict small molecules that potentially inhibit TIMELESS such as 8-azaguanine, gw8510, 6-thioguanosine, and ursodeoxycholic acid.

Conclusion: This study is the first comprehensive analysis of TIMELESS, revealing the relationship between the novel oncogene and the clinical characteristics of patients with glioma, and the mechanism leading to poor prognosis. It also provides a potential biomarker for the diagnosis and treatment of glioma. Our research hopes to reveal the pathological progress of glioma at the genetic level.

\section{Introduction}

Glioma is a kind of primary malignant tumor derived from neuroepithelial cells[1]. Its annual incidence rate accounts for about $1.6 \%$ of systemic tumors, but its mortality rate accounts for $2.5 \%$ of systemic tumors[2]. CT and MRI is currently the main assistant examination for the diagnosis of glioma[3, 4]. Patients with glioma in the middle and late stages often have typical imaging changes and a series of obvious dysfunctions[4]. Surgical treatment, adjuvant radiochemotherapy are the main means of clinical treatment. However, most patients after treatment, neurological dysfunction, and poor prognosis often bring huge economic and psychological burdens to society and families[5]. With the remarkable effect of CAR-T in treating leukemia, molecular targeted therapy has rekindled hope[6]. Therefore, in order to improve the treatment effect of glioma patients and restore the treatment confidence of the majority of patients, it is urgent to find new effective molecular markers.

At present, those special molecular markers discovered by scientists have been widely used in the early diagnosis of glioma and the personalized treatment of patients. For instance, Isocitrate dehydrogenase (IDH1), in low-grade glioma, mutations often show that the prognosis of IDH mutant is better than that of wild type[7]. Next, MGMT can promote the successful escape of tumor cells from alkylated chemotherapeutic drugs, resulting in patients being insensitive to drugs and having poor therapeutic effects[8]. In addition, Epidermal growth factor receptor (EGFR) participates in the formation of tumors 
through various channels, often related to the prognosis of glioblastoma[9, 10]. However, due to the diversity of glioma formation and the complexity of the tumor microenvironment, existing theories are difficult to fully reveal the specific pathogenesis of glioma[11]. Therefore, the discovery of new molecular markers will help to further reveal the new pathology of the glioma mechanism process and provide new molecular targets for evaluating patient prognosis and discovering new potential therapeutic drugs.

Cytogenetic and molecular genetic studies have shown that tumor development is a complex process with multiple factors and stages[12,13]. Genomic instability is considered to be a driving force in all stages of tumorigenesis[14]. TIMELESS participates in the maintenance of genome stability through multiple channels. For example, Anthony et al. found that TIMELESS can compile a conserved protein and gather it in the replication site of the nucleus, thereby contributing to DNA replication efficiency by combining Tipin[15]. Lauren and Si et al. found that TIMELESS migrated to DNA loss sites in a PARP1dependent manner, participated in the DNA repair process, and homologous recombination[16, 17]. Further research found that TIMELESS can also promote the malignant behavior of tumors and poor prognosis of patients. TIMELESS promotes the insensitivity of nasopharyngeal carcinoma to cisplatin through Wnt/b-catenin signaling pathway, thereby promoting tumor epithelial cell metastasis[18]. TIMELESS overexpression promotes the proliferation of colorectal cancer cells[19]. Qiu et al. found that overexpression of TIMELESS promotes poor prognosis in patients with kidney and liver cancer[20]. However, there is still little research about the role and mechanism of TIMELESS in glioma.

Our purpose is to use large samples in multiple databases to explain the correlation between TIMELESS expression and clinical features, and further explore the potential molecular mechanism of TIMELESS involvement in tumor formation and development. Therefore, we strongly believe that TIMELESS can be used as a potential molecular marker for clinical diagnosis and treatment and provides a new direction for revealing the pathological mechanism of glioma.

\section{Materials \& Methods}

\section{Data Extraction}

GEPIA (http://gepia.cancer-pku.cn/) is a well-known online tool for visual analysis of TCGA and GTEx data. Because of its simple interface, simple operation, and effective analysis, it is popular with researchers. Our study investigated the expression level of TIMELESS in various human tumor tissues and corresponding normal samples from this database. Among them, they include $163 \mathrm{GBM}$ samples, 518 LGG samples, and 207 normal brain tissues.

The Chinese Glioma Genome Atlas (CGGA) (http://www.cgga.org.cn/) is an open database that has been published for several years, especially used for glioma research and can provide a platform for researchers to analyze clinical information, data expression, gene copy, methylation[21]. Apart from missing data such as survival time, gender, and so on, out study obtained the RNA-seq of 748 glioma 
tissue samples and mRNA chip data of 268 glioma tissue samples, and for further analysis and processing. The filtered information is shown in Table S1 and Table S2.

The Tumor Genome Atlas (TCGA) ( http://cancergenome.nih.gov/) is a landmark public free database of cancer genomics, which contains many types of data such as genome, epigenome, transcriptome, and proteome. They have improved our ability to diagnose, treat, and prevent cancer and will continue to make it available to anyone in the research community[22]. After the deletion of missing clinical information data, we finally collected information on 653 tumor samples, seeing Table S3.

GEO (https://www.ncbi.nlm.nih.gov/geo/) is an open database serving the public, which is characterized by containing gene sequencing and chip data of multiple organisms based on multiple platforms[23]. It can provide a continuous data set so that researchers can systematically study the direction of interest. GSE4290 included 77 glioma and 23 normal brain tissue samples, GSE116520 included 34 glioma and 8 normal brain tissue samples and GSE50161 included 34 glioma and 13 normal brain tissue samples. We use the limma package in the $\mathrm{R}$ (version 3.6.1) language to analyze TIMELESS expression in glioma tissue and normal brain tissue[24](Fold Change value of $(|\log 2 \mathrm{FC}|)>1$ and $p$-value $<0.05)$.

The Human Protein Atlas (HPA) (http://www.proteinatlas.org/) is an open and free web tool that contains high-resolution images of millions of normal human tissues, cancer tissues, and human cell line proteins[25]. In order to obtain the difference in TIMELESS protein levels, we downloaded the TIMELESS immunohistochemistry results from the database and divided them into the normal group, high-grade glioma group, and low-grade glioma group.

\section{Gene Set Enrichment Analysis}

Gene Set Enrichment Analysis (GSEA) is used to evaluate the distribution trend of genes in a pre-defined gene set in the gene table sorted by phenotype correlation, so as to judge their contribution to phenotype[26]. We first standardize the processing of the original data from multiple databases (CGGA RNA-seq, CGGA microarray, and TCGA RNA-seq), and then divide the genes into two groups (high expression group, low expression group) according to the level of TIMELESS. GSEA 4.0.2 jar software was used in this study. The number of permutations was adjusted to 1000 , and the genome database was changed to the KEGG cell signaling pathway.

\section{Cmap}

The connectivity map (CMap) (https://cmap.ihmc.us/) helps us understand human diseases and accelerate the discovery of new therapies by creating and analyzing large perturbation data sets. We first use correlation analysis to obtain 10 genes positively related to TIMELESS and 10 genes negatively related. Then, we use the GPL570 platform to convert related genes into probe information, and then upload it to the CMap tool to analyze potential inhibitors of TIMELESS $(p<0.05$ and enrichment $<-0.88)$. 


\section{Statistical analysis}

The original data in this study were analyzed by Perl and R software. Wilcox test was analysed the expression of TIMELESS between tumor tissue and non-tumor tissue. We use the $\mathrm{R}$ language to calculate the relationship between TIMELESS and the survival time of glioma patients through the Cox regression and Kaplan-Meier method and draw a survival curve. Then, univariate and multivariate analysis reveals whether high expression of TIMELESS was an independent factor for patients' poor prognosis. Cox regression evaluates the value of TIMELESS as an independent factor. We discuss the relationship between clinical data and TIMELESS expression in glioma by using Wilcox or Kruskal test.

\section{Results}

\section{Abnormally high expression of TIMELESS in glioma.}

First, we analyzed the expression of TIMELESS in a variety of tumors and corresponding normal tissues through the GEPIA tool, and found that TIMELESS was widely high expression in BLCA, BRCA, CESC, COAD, DLBC, GBM, LGG, LUSC, OV, READ, SKCM, STAD, TGCT, THYM, UCEC, UCS, but was the low expression in LAML(Fig. 1A).

To further study the mechanism of TIMELESS in the tumor, we chose glioma as the research object. Through GSE4290 (77 glioma tissue samples and 23 normal brain tissue samples), GSE50161 (34 glioma tissue samples and 13 normal brain tissue samples), and GSE11652 (34 glioma tissue samples and 8 normal brain tissue samples) database analysis, we again verified that TIMELESS was indeed highly expressed in glioma (Fig. 1B-D, $p<0.001$ ). Through the mutual verification of multiple data sets, we can effectively improve the credibility of our data results. This study will lay a foundation for further study of the mechanism of TIMELESS in glioma.

\section{High expression of TIMELESS was an unfavorable factor for the prognosis of glioma patients.}

In order to evaluate the relationship between the high expression of TIMELESS and the prognosis of glioma patients, the original data from the CGGA RNA-seq, CGGA microarray, and TCGA RNA-seq databases were divided into a high expression group and a low expression group according to the expression of TIMELESS. Firstly, Compared with the low expression group of TIMELESS in 376 glioma patients through the Kaplan-Meier method, the high expression of TIMELESS in 372 glioma patients showed that the survival time of patients in the high expression group was significantly lower than that in the low expression group based on the CGGA RNA-seq (Fig. 2A). Secondly, Compared with the low expression group in 134 glioma patients through the Kaplan-Meier method, the high expression of TIMELESS in 134 glioma patients showed that TIMELESS can also reduce the survival time of glioma patients based on the CGGA microarray (Fig. 2B). Finally, in order to test the effect of TIMELESS expression in different ethnic groups on the prognosis of patients, we further verified the impact of high expression of TIMELESS on the prognosis of patients in TCGA RNA-seq, because the dataset mainly contains American race, while CGGA database contains Chinese race. It was found that the increased 
expression level of TIMELESS could significantly reduce the overall survival of glioma patients including 653 glioma patients (Fig. 2C). The results were surprisingly consistent with the CGGA database. However, whether high expression of TIMELESS was an independent factor for poor prognosis requires further study.

\section{High expression of TIMELESS was an independent factor for poor prognosis of glioma patients}

We used univariate and multivariate analysis to analyze the relationship between clinical sample information and the survival status of glioma patients. From the analysis of the common results in the above three databases, we found that TIMELESS expression and glioma grade is closely related to the prognosis of glioma patients (Fig. 3A-3F). The detailed results are as follows: in univariate analysis, TIMELESS expression and patient survival time have significant statistical significance in CGGA RNA-seq (HR: 1.733, 95\% Cl: 1.587-1.892, p<0.001, Fig. 3A), CGGA microarray (HR: 1.902; 95\% Cl: 1.636-2.212, $p$ $<0.001$, Fig. $3 C$ ) and TCGA RNA-seq (HR: 1.087, 95\% Cl: 1.064-1.109, $p<0.001$, Fig. 3E). In multivariate analysis, it also has statistical significance in CGGA RNA-seq (HR: 1.252, 95\% Cl: 1.137-1.379, $p<0.001$, Fig. 3B), CGGA microarray (HR: 1.483, 95\% Cl: 1.234-1.783, $p<0.001$, Fig. 3D) and TCGA RNA-seq (HR: $1.034,95 \% \mathrm{Cl}: 1.004-1.065, p<0.05$, Fig. 3F). At the same time, in univariate analysis, glioma grade and patient survival time have significant statistical significance in CGGA RNA-seq (HR: $2.883,95 \%$ Cl: $2.526-$ 3.291, $p<0.001$, Fig. 3A), CGGA microarray (HR: 2.567; 95\% Cl: 2.125-3.100, $p<0.001$, Fig. 3C) and TCGA RNA-seq (HR: 4.634, 95\% Cl:3.727-5.760, $p<0.001$, Fig. 3E). In multivariate analysis, it also has statistical significance in CGGA RNA-seq (HR: 2.506, 95\% Cl: 1.825-3.441, $p<0.001$, Fig. 3B), CGGA microarray (HR: $2.402,95 \% \mathrm{Cl}: 1.389-4.155, p<0.005$, Fig. 3D) and TCGA RNA-seq (HR: $3.044,95 \% \mathrm{Cl}$ : 2.401-3.860, $p<0.001$, Fig. 3F). But, due to incomplete clinical data in the TCGA RNA-seq database and the HR value of the age crosses the invalid line in the CGGA microarray multivariate analysis, whether PRS type and age can be used as an independent factor for glioma patients requires further analysis. In conclusion, TIMELESS High expression is an independent factor for poor prognosis of glioma patients.

\section{TIMELESS high expression has diagnostic value for patient prognosis}

To explore whether the high expression of TIMELESS has diagnostic value for the prognosis of glioma patients, we analyzed the above three sets of data and by cox regression. the high expression of TIMELESS as an independent factor for glioma patients with a poor prognosis has clinical diagnostic value in 1 year, 3 years, and 5 years from CGGA RNA-seq (Fig. 4A), CGGA microarray (Fig. 4B), and TCGA RNA-seq (Fig. 4C). The results suggest that TIMELESS can be used as a biomarker for the diagnosis and individualized treatment of glioma.

\section{The relationship between TIMELESS and clinical features of poor prognosis in patients with glioma.}

The results of Wilcox or Kruskal test correlation analysis showed that the abnormally high expression of TIMELESS was positively correlated with WHO grade, PRS type, chemo status, and histology (Fig. 5A, C, D, $\mathrm{G}, \mathrm{p}<0.001$ ), but IDH1 mutation and $1 p 19 q$ codeletion states were negatively correlated (Fig. $5 \mathrm{E}, \mathrm{F}, \mathrm{p}<$ 0.001) in the data of CGGA RNA-sEq. Meanwhile, it was positively correlated with WHO grade, histology, 
and negatively correlated with IDH1 mutation (Fig. 5A, E, G, p < 0.001) in CGGA microarray. Finally, it also was positively correlated with WHO grade, age in the data of TCGA RNA-seq (Fig. 5A, B, p < 0.001). By analyzing the above three databases, we found that the low expression of TIMELESS is closely related to low tumor grade, $1 p 19 q$ joint deletion, and IDH1 mutation. As our clinical knowledge knows, $1 p 19 q$ joint deletion, IDH1 mutations often occur in low-grade glioma, and patients often have a relatively good prognosis. Therefore, it further confirms indirectly supports that TIMELESS was a carcinogenic gene in glioma. However, the mechanism by which TIMELESS participates in the malignant behavior of glioma requires my next to reveal.

\section{Cell signaling pathways of TIMELESS in glioma}

We divided the tissue samples into the high expression group and low expression group based on the expression level of TIMELESS. Then, GSEA analysis was used to reveal the activation of cancer-related cell signaling pathways caused by high expression of TIMELESS. Potential cellular molecular pathways were selected out by the consistent results of three databases together enriched (Table 1). The TIMELESS high expression may participate in tumor development by activating cell cycle, mismatch repair, and DNA replication cell signaling pathways (Fig. 6A-C).

Table 1

The gene set enriches the high TIMELESS in three databases.

\begin{tabular}{|lllllll|}
\hline GENE SET NAME & \multicolumn{2}{l}{ CGGA RNA-seq } & \multicolumn{2}{l|}{ CGGA microarray } & \multicolumn{2}{l|}{ TCGA RNA-seq } \\
\cline { 2 - 8 } & $\begin{array}{l}\text { NOM p- } \\
\text { value }\end{array}$ & $\begin{array}{l}\text { FDR q- } \\
\text { value }\end{array}$ & $\begin{array}{l}\text { NOM p- } \\
\text { value }\end{array}$ & $\begin{array}{l}\text { FDR q- } \\
\text { value }\end{array}$ & $\begin{array}{l}\text { NOM p- } \\
\text { value }\end{array}$ & $\begin{array}{l}\text { FDR q- } \\
\text { value }\end{array}$ \\
\hline CELL CYCLE & 0.000 & 0.061 & 0.000 & 0.000 & 0.000 & $7.52 E-04$ \\
\hline DNA REPLICATION & 0.000 & 0.108 & 0.000 & 0.018 & 0.000 & 0.005 \\
\hline MISMATCH REPAIR & 0.002 & 0.113 & 0.008 & 0.043 & 0.000 & 0.005 \\
\hline BASE EXCISION & 0.002 & 0.203 & 0.039 & 0.125 & 0.000 & 0.005 \\
\hline $\begin{array}{l}\text { HOMOLOGOUS } \\
\text { RECOMBINATION }\end{array}$ & 0.006 & 0.180 & 0.000 & $4.00 E-04$ & 0.000 & 0.005 \\
\hline $\begin{array}{l}\text { Gene sets with NOM P-value } \\
\text { NOM: nominal; FDR: false discovery rate. }\end{array}$ & & & & & \\
\hline
\end{tabular}

\section{Abnormally high expression of TIMELESS protein levels in glioma}

We have verified the abnormally high expression of TIMELESS in glioma from the level of mRNA. However, the level of TIMELESS protein is unclear. So, we downloaded the immunohistochemical film of TIMELESS protein expression in glioma tissue and normal brain tissue from The Human Protein Atlas website. Then divided into three groups according to tumor grade, including the normal group, low-grade group, high-grade group. We found that the TIMELESS protein expression level was markedly higher in both the low-level group and the high-level group than the normal group (Fig. 7). 


\section{Drugs of potential inhibit TIMELESS}

In order to find drugs that potentially inhibit expression of TIMELESS, we first performed a correlation analysis between TIMELESS and other differential genes. Select 10 positively related genes and 10 negatively related genes and draw a circle diagram (Fig. 8). Next, we uploaded these 20 related genes to the CMap website and screened out 4 small molecule compounds that potentially inhibit TIMELESS, such as 8-azaguanine, gw8510, 6-Thioguanosine, and ursodeoxycholic acid (Fig. 9, Table 2). Some of these drugs have been found to have clear anti-tumor effects in other studies. This reflects the reliability of our predictions.

Table 2

Small molecule compounds predicted by CMap

\begin{tabular}{|llll|}
\hline NO. & CMap name & enrichment & $\mathbf{p}$ \\
\hline 1 & GW-8510 & -0.929 & 0.00002 \\
\hline 2 & roxithromycin & -0.904 & 0.00016 \\
\hline 3 & ursodeoxycholic acid & -0.885 & 0.003 \\
\hline 4 & thioguanosine & -0.882 & 0.00048 \\
\hline 5 & 8-azaguanine & -0.786 & 0.00426 \\
\hline \multicolumn{4}{|l}{ Enrichment<-0.7, $\mathrm{p}<0.05$. connectivity map: CMap } \\
\hline
\end{tabular}

\section{Discussion}

In recent years, a large number of studies have shown that TIMELESS can affect the process of tumor formation through proliferation, migration, and inhibition of apoptosis[19, 27, 28]. However, the effect of TIMELESS on the prognosis of glioma patients and its molecular mechanism has not been elucidated. We use the advantages of large samples to reveal the role of TIMELESS in glioma. From mRNA to protein levels, we found that TIMELESS was highly expressed in glioma relative to normal brain tissues (Fig. 1, Fig. 7). At the same time, our conclusions are mutually verified with previous studies. For example, Bianco found that TIMELESS was abnormally highly expressed in non-small-cell lung cancers (NSCLC) and breast cancers[29]. Zhou confirmed TIMELESS expression was higher in cervical cancer from both public databases and clinical samples[30]. Wang also verified that TIMELESS was highly expressed in glioma[31]. However, the study did not elaborate on the relationship between TIMELESS and the clinical characteristics of glioma patients, and the underlying molecular mechanism.

We firstly found TIMELESS was associated with the prognosis of glioma patients. As described in Fig. 24, high expression of TIMELESS was an independent risk factor for the prognosis of glioma patients and has clinical diagnostic value. In other tumors, we also found that TIMELESS is closely related to clinical prognosis. For instance, TIMELESS in lung cancer promotes the malignant characteristics of tumors and leads to a poor prognosis for patients[32]. Zhang et al. found that TIMELESS promotes the malignant 
progression of tumors and poor prognosis in cervical carcinoma[33]. Just like the result of Fig. 5, it indicates that the expression level of TIMELESS was positively correlated with the age of patients and the grade of glioma. As we all know, grade $₫-\otimes$ glioma show diffuse infiltrating growth with high heterogeneity. The postoperative radiotherapy and chemotherapy were often resistant, and the patient's prognosis was less than ideal[34]. It is completely consistent with our point of view in this experiment. Therefore, we speculate that the high expression of TIMELESS was mainly involved in the malignant behavior in the late stage of the tumor, which provides a direction for the next step to explore the specific molecular mechanism of TIMELESS in the tumor.

We used GSEA to analyze the three databases and found that the high expression of TIMELESS may participate in the malignant progression of the tumor through the cell cycle, mismatch repair, and DNA replication cell signaling pathways. GSEA and enrichment analysis (GO analysis and Pathway analysis) are two commonly used to predict the molecular mechanism of participation. Enrichment analysis often focuses on a part of significantly different gene groups, which is easy to miss some genes that are not significantly differentially expressed but have important biological significance[35]. GSEA does not need to set the threshold of differential genes in advance and analyze several genes from the expression profile level according to the overall trend of actual data[36]. In addition, our predicted pathway has been reported to be associated with tumorigenicity. Emmanuel found that mismatch repair genes mutations in ovarian cancer can promote the growth of tumor cells to a distance and worsen the clinical physiology of patients[37]. Kristin discovered that the excision repair system was abnormally high in the early stage of many tumor cells and promotes tumor malignant progression[38]. Evan found that the process of tumor formation was diverse, but the cell cycle was a part that cannot be ignored[39]. Therefore, through the above description, we can draw a general conclusion that the abnormal increase of TIMELESS in glioma may lead to poor prognosis through a variety of cell signaling pathways.

Using the advantages of public databases, we have demonstrated that the high expression of TIMELESS was closely related to the malignant behavior of high-grade glioma. However, there are some flaws in this experiment. First, the clinical information of some samples in the database was incomplete, the treatment plan was not clear and the database also has a regional. We use a combination of multiple databases to expand the sample size to make up for the problem and avoid sample bias. Second, the cell signaling pathway of GSEA analysis did not prove with traditional experiments. But, the GSEA method has been reported in many articles and has certain feasibility. Besides, TIMELESS may have other effects and this article cannot specifically reveal all the pathogenesis.

\section{Conclusion}

The high expression of TIMELESS was closely related to the prognosis of glioma patients and can be used as an independent prognostic factor in the clinic. It can also be used as a molecular marker for screening with glioma and provides a potential target for gene therapy of glioma.

\section{Declarations}




\section{Ethics approval and consent to participate}

All of our research data are from public databases and do not involve ethical issues.

\section{Competing interests}

The authors declare that they have no competing interests.

\section{Funding}

The authors received no funding for this work

\section{Authors' contributions}

ZDL, ZSR and ZBH were major contributors in writing the manuscrip and designed the study, XBC and WZ performed the literature search, BFL and JLW revised the manuscript, YQG and YZG made a critical improvement on the experiment and manuscript. All authors read and approved the final manuscript.

\section{Acknowledgements}

Not applicable.

\section{References}

1. Lapointe S, Perry A, Butowski N. Primary brain tumours in adults. Lancet. 2018;392(10145):432-46.

2. Bray F, Ferlay J, Soerjomataram I, et al. Global cancer statistics 2018: GLOBOCAN estimates of incidence and mortality worldwide for 36 cancers in 185 countries. Cancer J Clin. 2018;68(6):394424.

3. Ellingson B, Wen P, Cloughesy T. Evidence and context of use for contrast enhancement as a surrogate of disease burden and treatment response in malignant glioma. Neurooncology. 2018;20(4):457-71.

4. Omuro A, DeAngelis L. Glioblastoma and other malignant gliomas: a clinical review. JAMA. 2013;310(17):1842-50.

5. Sturm D, Pfister S, Jones D. Pediatric Gliomas: Current Concepts on Diagnosis, Biology, and Clinical Management. Journal of clinical oncology: official journal of the American Society of Clinical Oncology. 2017;35(21):2370-7.

6. Guedan S, Ruella M, June C. Emerging Cellular Therapies for Cancer. Annu Rev Immunol. 2019;37:145-71. 
7. Gusyatiner O, Hegi M. Glioma epigenetics: From subclassification to novel treatment options. Sem Cancer Biol. 2018;51:50-8.

8. Chen R, Smith-Cohn M, Cohen A, et al. Glioma Subclassifications and Their Clinical Significance. Neurotherapeutics: the journal of the American Society for Experimental NeuroTherapeutics. 2017;14(2):284-97.

9. Mercer R, Tyler M, Ulasov I, et al. Targeted therapies for malignant glioma: progress and potential. BioDrugs: clinical immunotherapeutics, biopharmaceuticals and gene therapy. 2009;23(1):25-35.

10. Saadeh F, Mahfouz R, Assi H. EGFR as a clinical marker in glioblastomas and other gliomas. Int J Biol Mark. 2018;33(1):22-32.

11. Hanahan D, Weinberg R. Hallmarks of cancer: the next generation. Cell. 2011;144(5):646-74.

12. Goussia A, Agnantis N, Rao J, et al. Cytogenetic and molecular abnormalities in astrocytic gliomas (Review). Oncol Rep. 2000;7(2):401-12.

13. Joo M, Park S, Chang $S$, et al. Cytogenetic and molecular genetic study on granular cell glioblastoma: a case report. Human pathology. 2013;44(2):282-8.

14. Meeker A, Argani P. Telomere shortening occurs early during breast tumorigenesis: a cause of chromosome destabilization underlying malignant transformation? J Mammary Gland Biol Neoplasia. 2004;9(3):285-96.

15. Gotter A, Suppa C, Emanuel B. Mammalian TIMELESS and Tipin are evolutionarily conserved replication fork-associated factors. Journal of molecular biology. 2007;366(1):36-52.

16. Young L, Marzio A, Perez-Duran P, et al. TIMELESS Forms a Complex with PARP1 Distinct from Its Complex with TIPIN and Plays a Role in the DNA Damage Response. Cell reports. 2015;13(3):451-9.

17. Xie S, Mortusewicz O, Ma H, et al. Timeless Interacts with PARP-1 to Promote Homologous Recombination Repair. Molecular cell. 2015;60(1):163-76.

18. Liu S, Lin H, Lin C, et al. TIMELESS confers cisplatin resistance in nasopharyngeal carcinoma by activating the $\mathrm{Wnt} / \beta$-catenin signaling pathway and promoting the epithelial mesenchymal transition. Cancer letters. 2017;402:117-30.

19. Neilsen B, Frodyma D, McCall J, et al. ERK-mediated TIMELESS expression suppresses G2/M arrest in colon cancer cells. PloS one. 2019;14(1):e0209224.

20. Qiu M, Liu L, Jin S, et al. Research on circadian clock genes in common abdominal malignant tumors. Chronobiology international. 2019;36(7):906-18.

21. Liu Z, Shen F, Wang H, et al. Abnormally high expression of HOXA2 as an independent factor for poor prognosis in glioma patients. Cell cycle (Georgetown Tex). 2020;19(13):1632-40.

22. Colaprico A, Silva T, Olsen $C$, et al. TCGAbiolinks: an R/Bioconductor package for integrative analysis of TCGA data. Nucleic acids research. 2016;44(8):e71.

23. Barrett T, Wilhite $S$, Ledoux P, et al. NCBI GEO: archive for functional genomics data sets-update. Nucleic acids research. 2013;41:D991-5. 
24. Liu Y, Niu R, Li W, et al. Therapeutic potential of menstrual blood-derived endometrial stem cells in cardiac diseases. Cell Mol Life Sci. 2019;76(9):1681-95.

25. Pontén F, Jirström K, Uhlen M. The Human Protein Atlas-a tool for pathology. J Pathol. 2008;216(4):387-93.

26. Subramanian A, Tamayo P, Mootha V, et al. Gene set enrichment analysis: a knowledge-based approach for interpreting genome-wide expression profiles. Proc Natl Acad Sci USA. 2005;102(43):15545-50.

27. Zou X, Zhu C, Zhang L, et al. MicroRNA-708 Suppresses Cell Proliferation and Enhances Chemosensitivity of Cervical Cancer Cells to cDDP by Negatively Targeting Timeless. OncoTargets therapy. 2020;13:225-35.

28. Elgohary N, Pellegrino R, Neumann O, et al. Protumorigenic role of Timeless in hepatocellular carcinoma. Int J Oncol. 2015;46(2):597-606.

29. Bianco J, Bergoglio V, Lin Y, et al. Overexpression of Claspin and Timeless protects cancer cells from replication stress in a checkpoint-independent manner. Nature communications. 2019;10(1):910.

30. Zhou J, Zhang Y, Zou X, et al. Aberrantly Expressed Timeless Regulates Cell Proliferation and Cisplatin Efficacy in Cervical Cancer. Human gene therapy. 2020;31:385-95.

31. Wang F, Chen Q. timelessThe analysis of deregulated expression of the genes in gliomas. J Cancer Res Ther. 2018;14:708-S12.

32. Yoshida $\mathrm{K}$, Sato $\mathrm{M}$, Hase $\mathrm{T}$, et al. TIMELESS is overexpressed in lung cancer and its expression correlates with poor patient survival. Cancer Sci. 2013;104(2):171-7.

33. Zhang W, He W, Shi Y, et al. Aberrant TIMELESS expression is associated with poor clinical survival and lymph node metastasis in early-stage cervical carcinoma. Int J Oncol. 2017;50(1):173-84.

34. Tan A, Ashley D, López G, et al. Management of glioblastoma: State of the art and future directions. Cancer J Clin. 2020;70(4):299-312.

35. Zhang $C$, Peng $L$, Zhang $Y$, et al. The identification of key genes and pathways in hepatocellular carcinoma by bioinformatics analysis of high-throughput data. Medical oncology (Northwood, London, England). 2017;34(6):101.

36. Zhang Y, Topham D, Thakar J, et al. FUNNEL-GSEA: FUNctioNal ELastic-net regression in time-course gene set enrichment analysis. Bioinformatics. 2017;33(13):1944-52.

37. Antonarakis E, Shaukat F, Isaacsson Velho P, et al. Clinical Features and Therapeutic Outcomes in Men with Advanced Prostate Cancer and DNA Mismatch Repair Gene Mutations. European urology. 2019;75(3):378-82.

38. Limpose K, Trego K, Li Z, et al. Overexpression of the base excision repair NTHL1 glycosylase causes genomic instability and early cellular hallmarks of cancer. Nucleic acids research. 2018;46(9):451532.

39. Evan G, Vousden K. Proliferation, cell cycle and apoptosis in cancer. Nature. 2001;411(6835):342-8. 
Figures

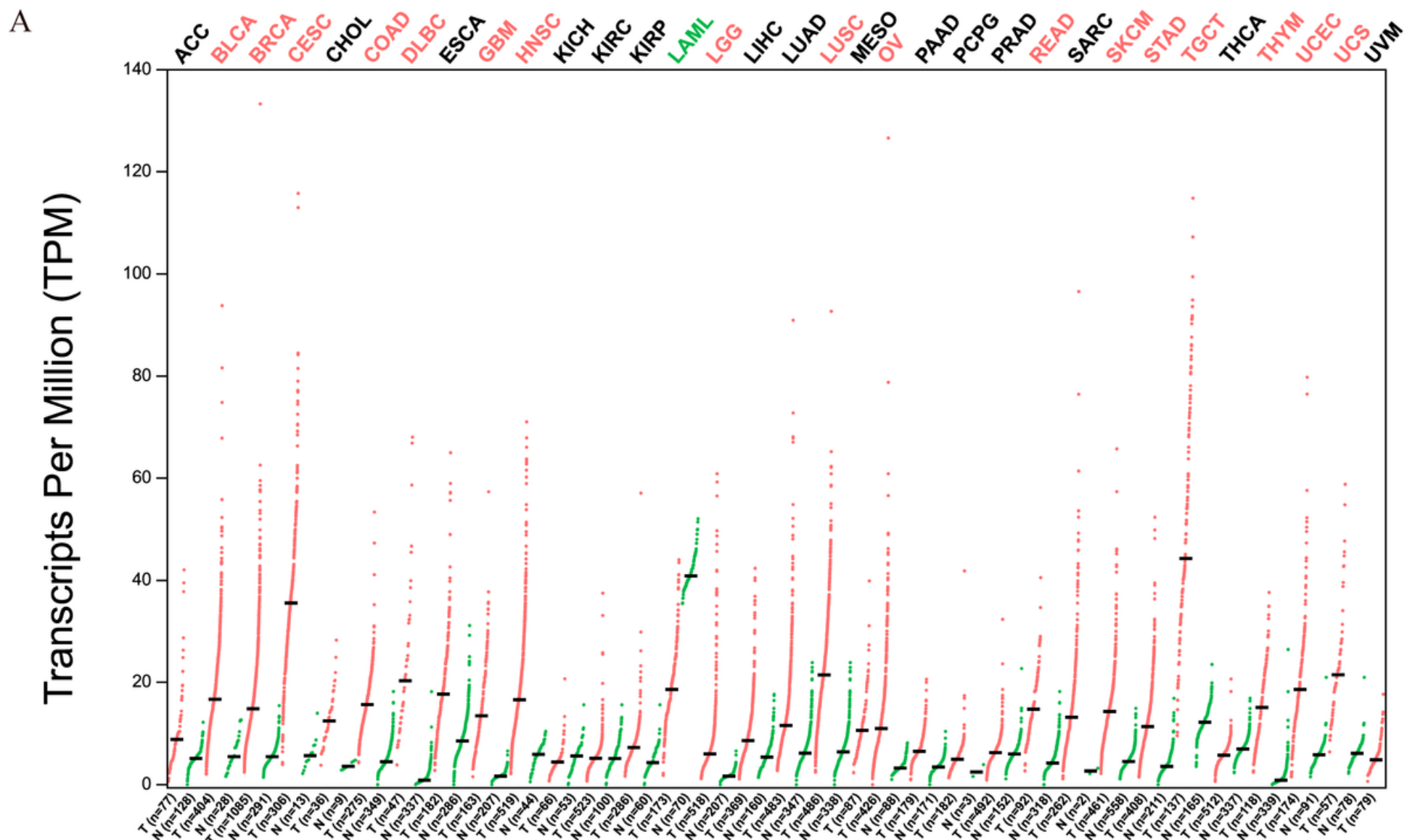

$\mathrm{B}$

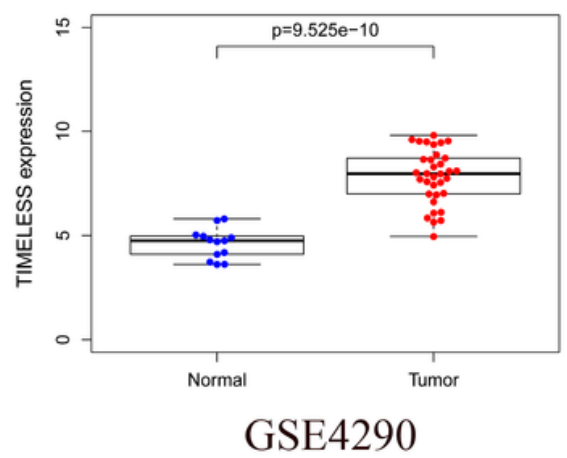

$\mathrm{C}$

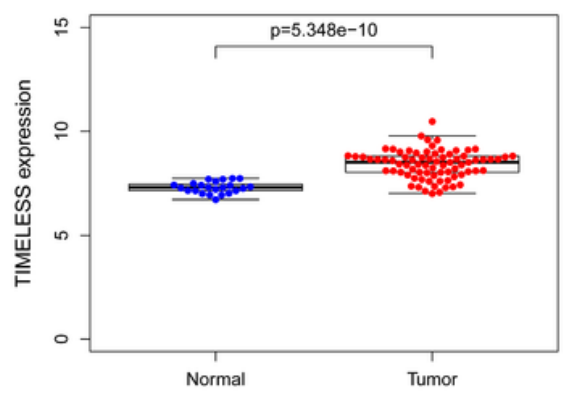

GSE50161
$\mathrm{D}$

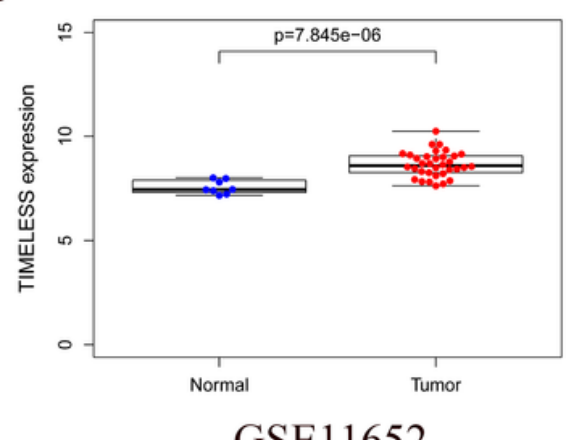

GSE11652

Figure 1

The expression level changes of TIMELESS in glioma. (A): TIMELESS is a widely expressed in a variety of tumors: red indicates cancer with up-regulation of TIMELESS, while green indicates a decrease in the expression of TIMELESS in malignant tissues. (B-D): TIMELESS expression is higher in glioma than normal brain tissues from GEO (GSE4290, GSE50161, GSE116520, $p<0.001$ ). 
A

TMELESS lovel to nigh to low
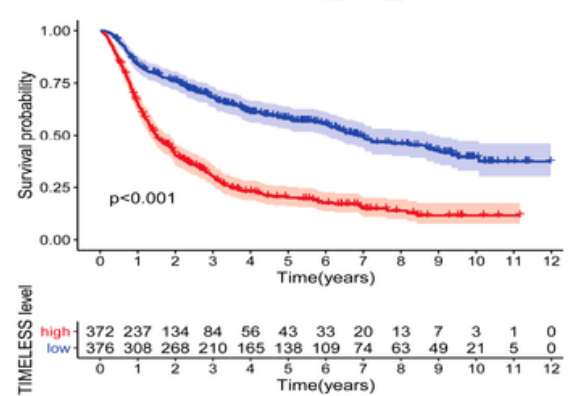

CGGA RNA-seq
B



CGGA microarray
$\mathrm{C}$
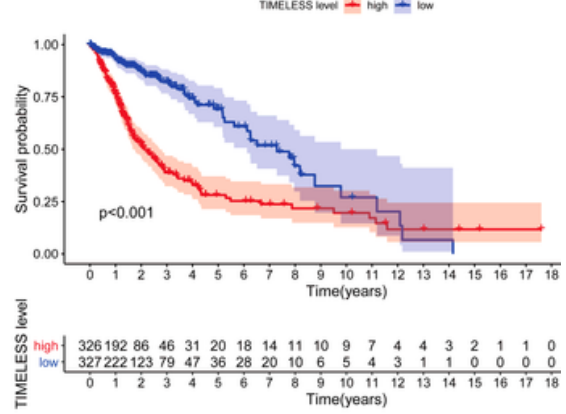

TCGA RNA-seq

\section{Figure 2}

Correlation analysis of TIMELESS and survival time of patients based on multiple databases. (A): in CGGA RNA-seq; (B): in CGGA microarray; (C): in TCGA RNA-seq. p<0.05. CGGA RNA-seq: the Chinese Glioma Genome Atlas RNA- Sequence, CGGA microarray: the Chinese Glioma Genome Atlas microarray, TCGA RNA-seq: The Cancer Genome Atlas RNA- Sequence. 
A

$\begin{array}{lrr} & \text { pvalue } & \begin{array}{r}\text { Hazard ratio } \\ \text { TIMELESS }\end{array} \\ <0.001 & 1.733(1.587-1.892) \\ \text { PRS_type } & <0.001 & 2.123(1.818-2.478) \\ \text { Histology } & <0.001 & 4.487(3.695-5.449) \\ \text { Grade } & <0.001 & 2.883(2.526-3.291) \\ \text { Gender } & 0.655 & 1.044(0.866-1.258) \\ \text { Age } & <0.001 & 1.624(1.345-1.960) \\ \text { Radio } & 0.571 & 0.929(0.720-1.199) \\ \text { Chemo } & <0.001 & 1.647(1.328-2.044) \\ \text { IDH_mutation } & <0.001 & 0.317(0.262-0.384) \\ \text { 1p19q_codeletion } & <0.001 & 0.231(0.169-0.315)\end{array}$



CGGA RNA-seq(univariate)

C



CGGA microarray(univariate)

E

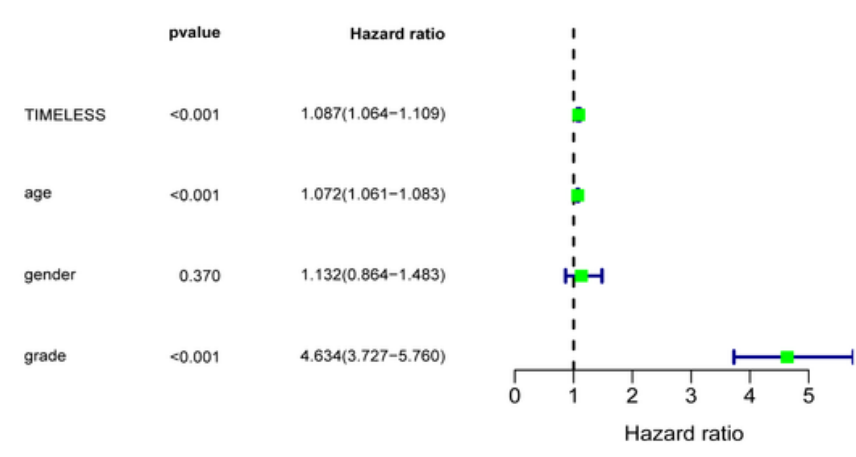

TCGA RNA-seq(univariate)
B

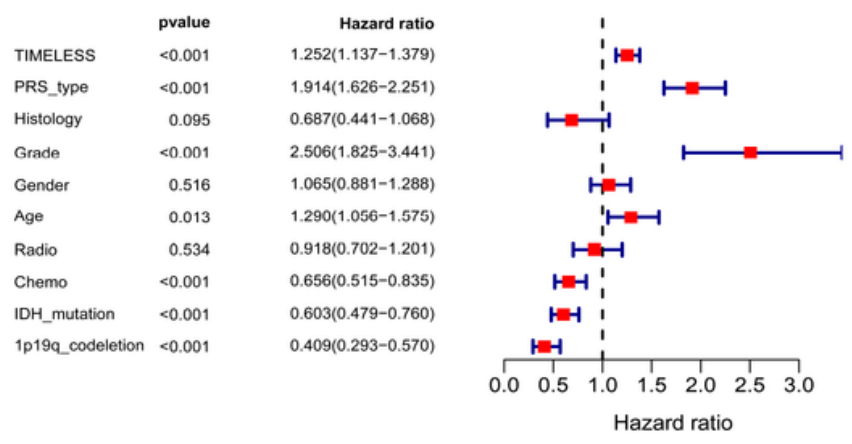

D

CGGA RNA-seq(multivariate)

$\begin{array}{lrr} & \text { pvalue } & \text { Hazard ratio } \\ \text { TIMELESS } & <0.001 & 1.483(1.234-1.783) \\ \text { TCGA_subtypes } & 0.078 & 0.859(0.725-1.017) \\ \text { PRS_type } & 0.004 & 1.607(1.165-2.217) \\ \text { Histology } & 0.360 & 0.683(0.302-1.545) \\ \text { Grade } & 0.002 & 2.402(1.389-4.155) \\ \text { Gender } & 0.893 & 0.979(0.713-1.343) \\ \text { Age } & 0.173 & 1.256(0.905-1.742) \\ \text { Radio } & 0.035 & 0.570(0.339-0.960) \\ \text { Chemo } & 0.743 & 0.944(0.667-1.335) \\ \text { IDH_mutation } & 0.439 & 0.856(0.578-1.268)\end{array}$

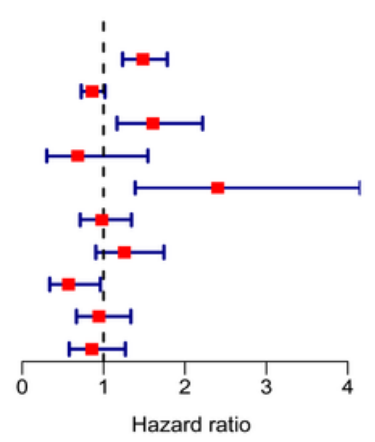

CGGA microarray(multivariate)

F


TCGA RNA-seq(multivariate)

\section{Figure 3}

TIMELESS is an independent factor of poor prognosis in glioma through univariate and multivariate analysis. (A, C, E) are univariate analysis from CGGA RNA-seq, CGGA microarray and TCGA RNA-seq. (B, $D, F)$ are multivariate analysis from CGGA RNA-seq, CGGA microarray and TCGA RNA-seq. 
A

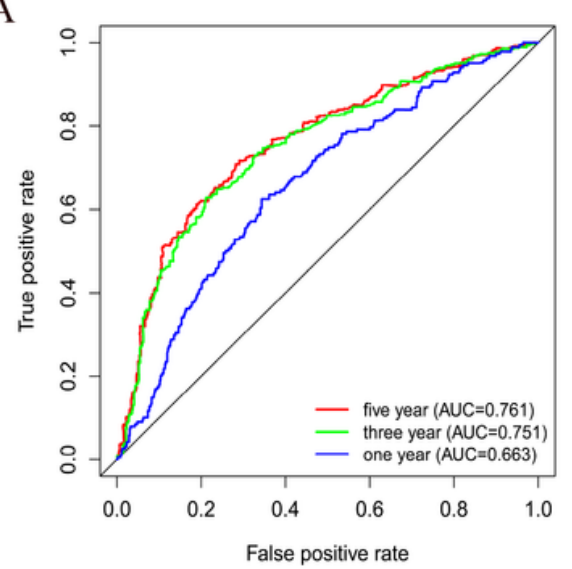

CGGA RNA-seq
B

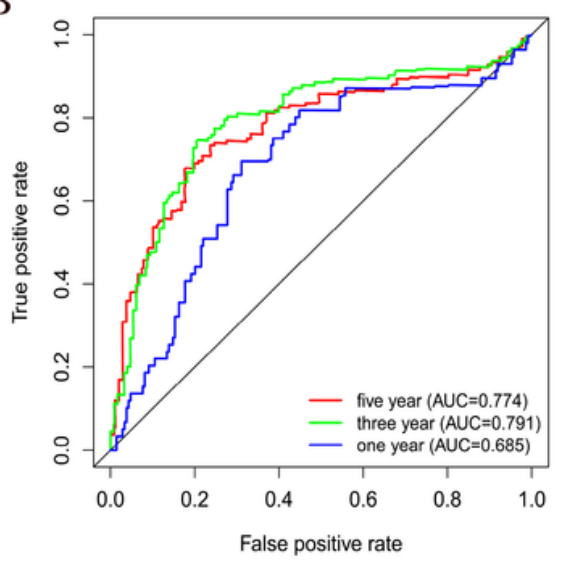

CGGA microarray



TCGA RNA-seq

Figure 4

TIMELESS has certain clinical diagnostic value through ROC curve analysis. (A): in CGGA RNA-seq; (B): in CGGA microarray; (C) in TCGA RNA-seq. 
A WHO grade

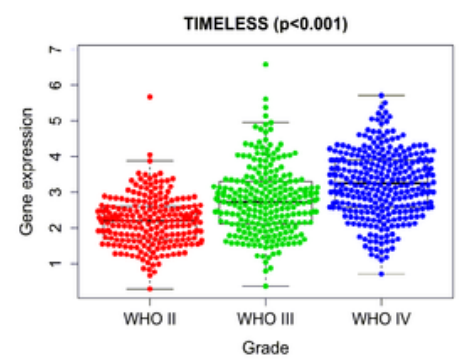

CGGA RNA-seq



CGGA microarray

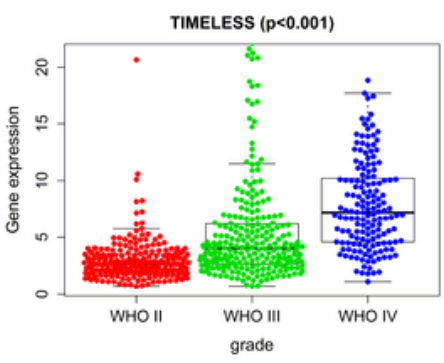

TCGA RNA-seq
B Age



TCGA RNA-seq

E IDH Mutation status

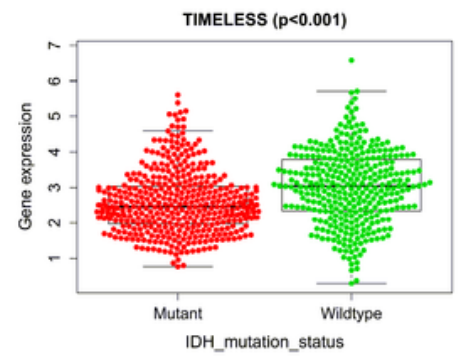

CGGA RNA-seq
C PRS type

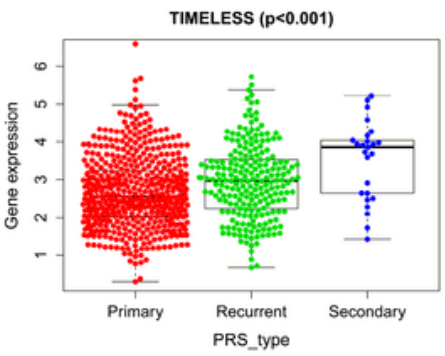

CGGA RNA-seq

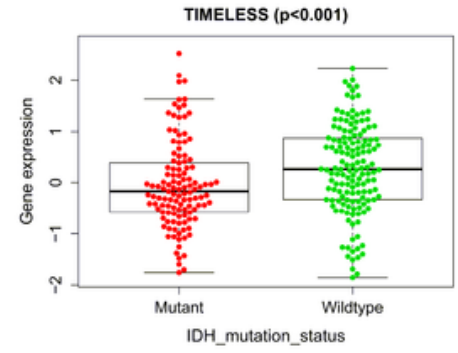

CGGA microarray
D Chemo status

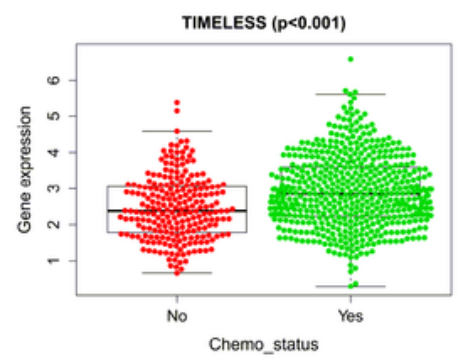

CGGA RNA-seq

F $1 \mathrm{p} 19 \mathrm{q}$ codeletion status

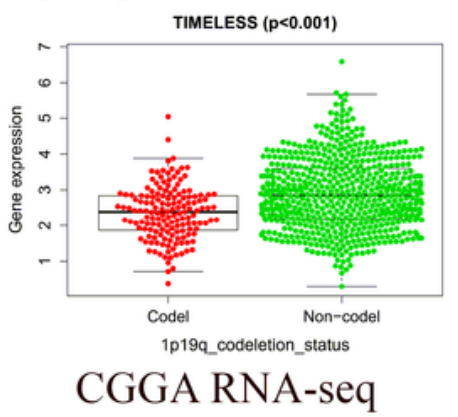

\section{G Histology}

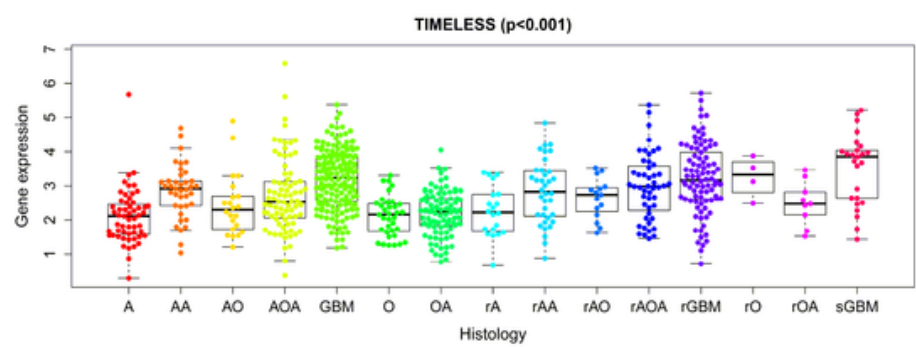

CGGA RNA-seq



CGGA microarray

\section{Figure 5}

Correlation analysis of TIMELESS and clinical features in CGGA RNA-seq, CGGA microarray and TCGA RNA-seq datasets. A: WHO Grade; B: age; C: PRS type; D: Chemo Status; E: IDH Mutation Status; F: 1p19q codeletion status; G: histology. PRS: post-resuscitation syndrome, IDH: Isocitrate dehydrogenase. 
A

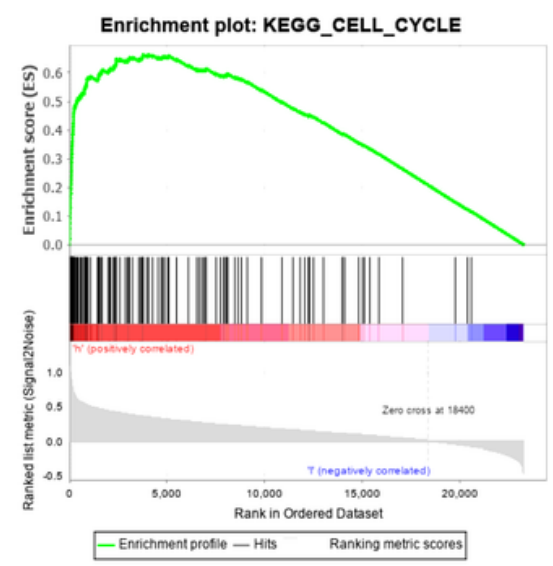

CGGA RNA-seq

B

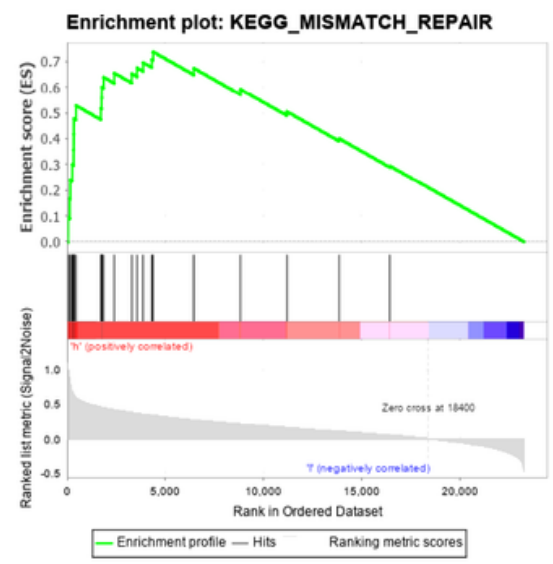

CGGA RNA-seq

C

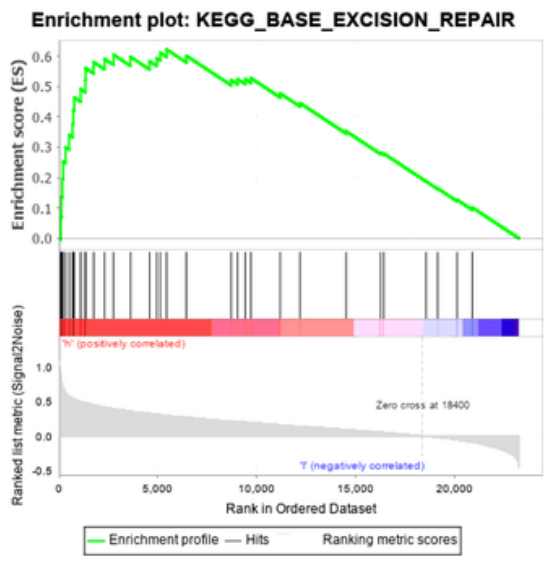

CGGA RNA-seq

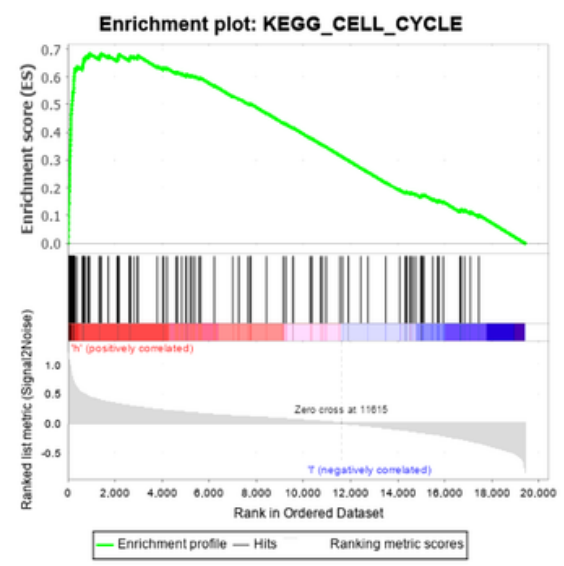

CGGA microrray

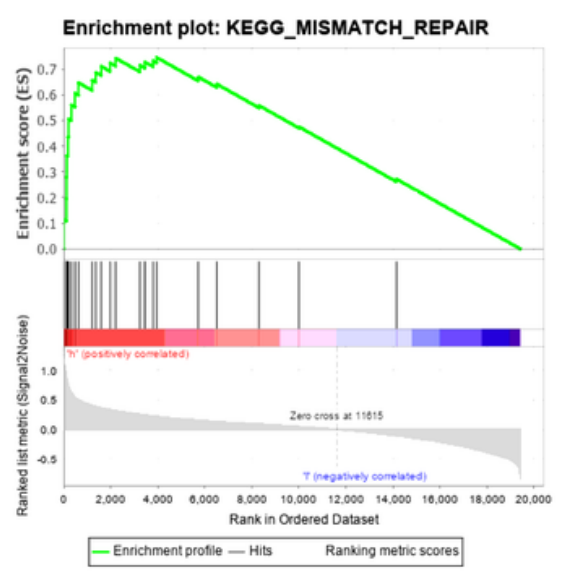

CGGA microarray

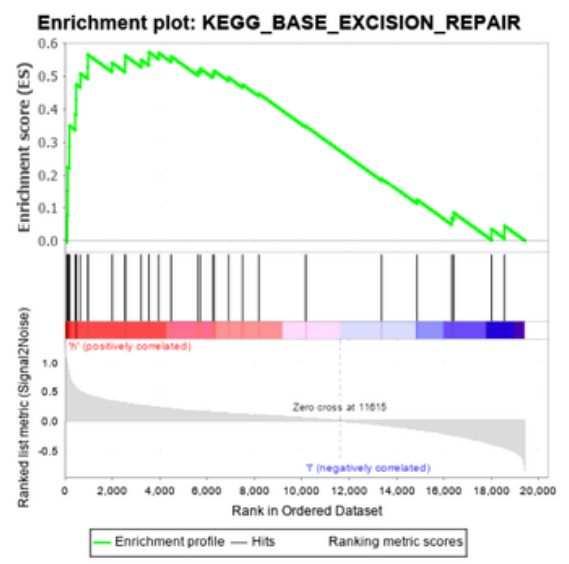

CGGA microarray



TCGA RNA-seq

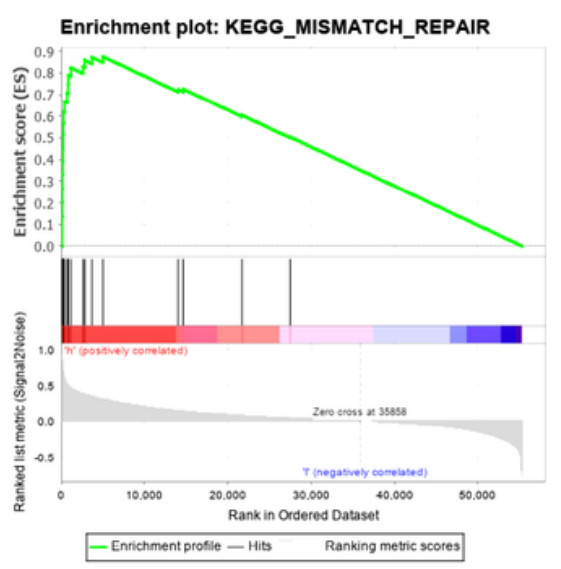

TCGA RNA-seq

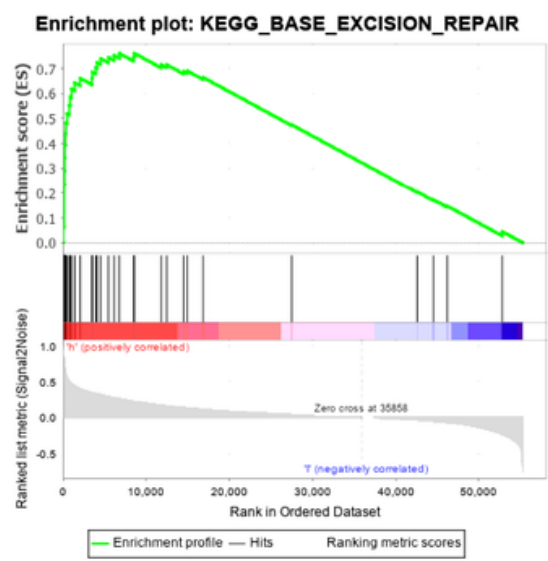

TCGA RNA-seq

Figure 6

Enrichment of TIMELESS potential cell signaling pathways through GSEA. (A): CELL CYCLE signaling pathway; (B): MISMATCH REPAIR signaling pathway; (C): BASE EXCISION signaling pathway. EC: Enrichment score. 
A

F 42 ID: 4626

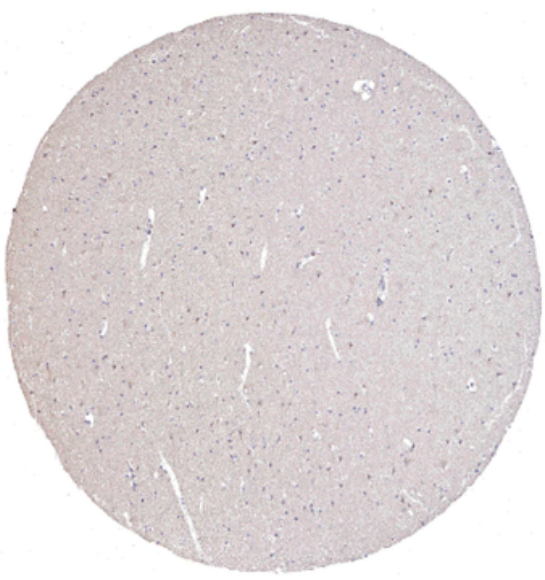

D $\quad$ M 37 ID: 3731

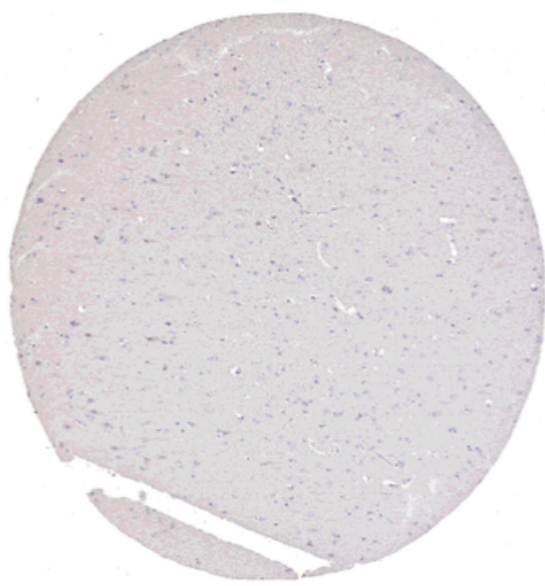

Normal
B

F 32 ID: 122

C $\quad$ M 49 ID: 2891

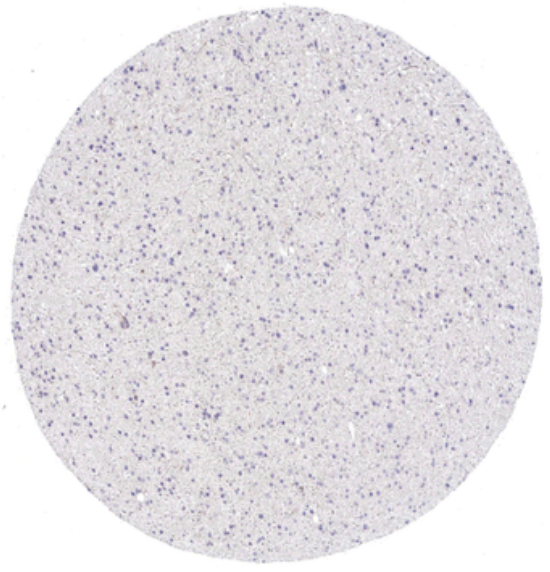

E $\quad$ M 66 ID: 2895

F $\quad$ M 76 ID: 2907

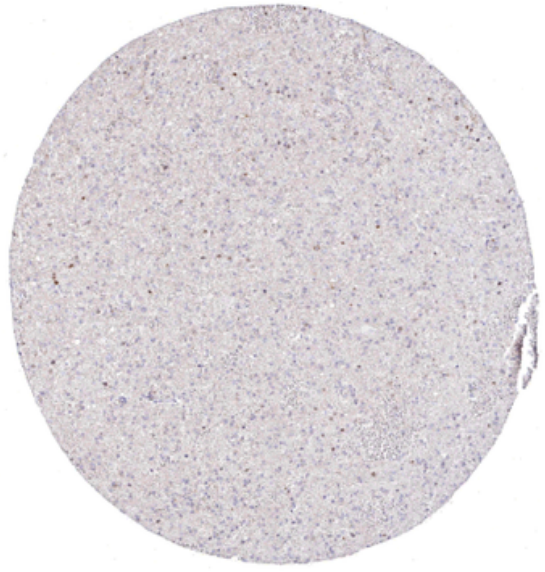

Low grade glioma


High grade glioma

Figure 7

Expression of TIMELESS protein from glioma tissue and normal brain tissue. $(A, B)$ : in normal brain tissue; $(C, D)$ : in low grade glioma tissue; $(E, F)$ : in high grade glioma tissue. $M$ : male, F: female. ID from ATLAS. 


\begin{tabular}{|c|c|c|}
\hline Gene & Cor & pvalue \\
\hline FBXW4 & -0.487 & $8.17 \mathrm{E}-62$ \\
\hline MATK & -0.486 & $2.49 \mathrm{E}-61$ \\
\hline PNMAL2 & -0.486 & $1.88 \mathrm{E}-61$ \\
\hline PHYHIP & -0.477 & $4.61 \mathrm{E}-59$ \\
\hline TMEM56 & -0.468 & $1.38 \mathrm{E}-56$ \\
\hline SCN2B & -0.46 & $1.48 \mathrm{E}-54$ \\
\hline LDHD & -0.459 & $3.66 \mathrm{E}-54$ \\
\hline AC090425.1 & -0.454 & $6.82 \mathrm{E}-53$ \\
\hline SNAI3-AS1 & -0.446 & $5.69 \mathrm{E}-51$ \\
\hline GRIN1 & -0.441 & $9.96 \mathrm{E}-50$ \\
\hline NCAPH & 0.881 & $0.00 \mathrm{E}+00$ \\
\hline CENPF & 0.878 & $0.00 \mathrm{E}+00$ \\
\hline ZWINT & 0.875 & $1.18575755001899 \mathrm{e}-322$ \\
\hline NUF2 & 0.874 & $1.87547319161337 \mathrm{e}-320$ \\
\hline GINS1 & 0.869 & $3.12742233703747 \mathrm{e}-313$ \\
\hline GTSE1 & 0.869 & $1.81474210735347 \mathrm{e}-313$ \\
\hline MLF1IP & 0.866 & $3.97 \mathrm{E}-308$ \\
\hline NUSAP1 & 0.866 & $1.56 \mathrm{E}-307$ \\
\hline CDK2 & 0.865 & $1.88 \mathrm{E}-306$ \\
\hline MELK & 0.865 & $1.40 \mathrm{E}-306$ \\
\hline BUB1 & 0.862 & $7.00 \mathrm{E}-302$ \\
\hline
\end{tabular}

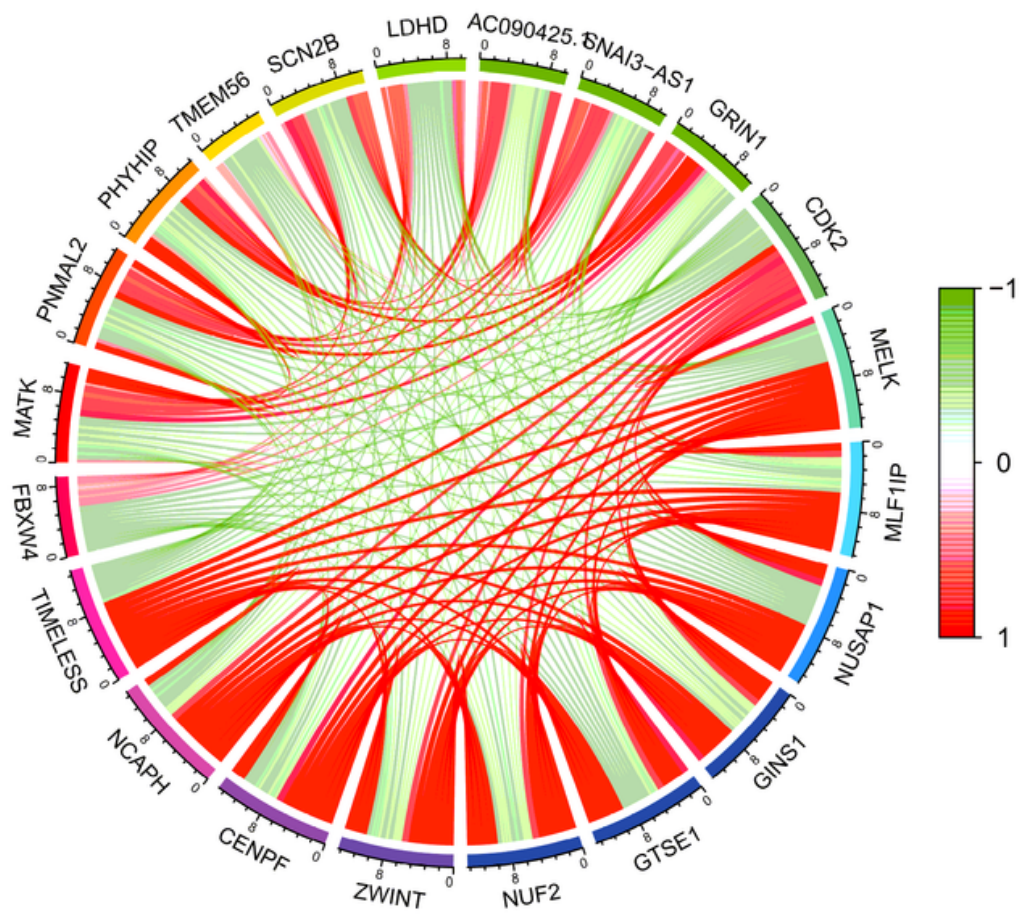

Figure 8

Correlation analysis between TIMELESS and other differential genes. (A, B): Screen out the top 10 differential genes with positive and negative correlations. 
A


Gw8510

$\mathrm{C}_{21} \mathrm{H}_{15} \mathrm{~N}_{5} \mathrm{O}_{3} \mathrm{~S}_{2}$

Compound CID: 3536

B

<smiles>Nc1nc2n[nH]nc2c(=O)[nH]1</smiles>

\section{8-Azaguanine}

$\mathrm{C}_{4} \mathrm{H}_{4} \mathrm{~N}_{6} \mathrm{O}$

Compound CID:135403646

C
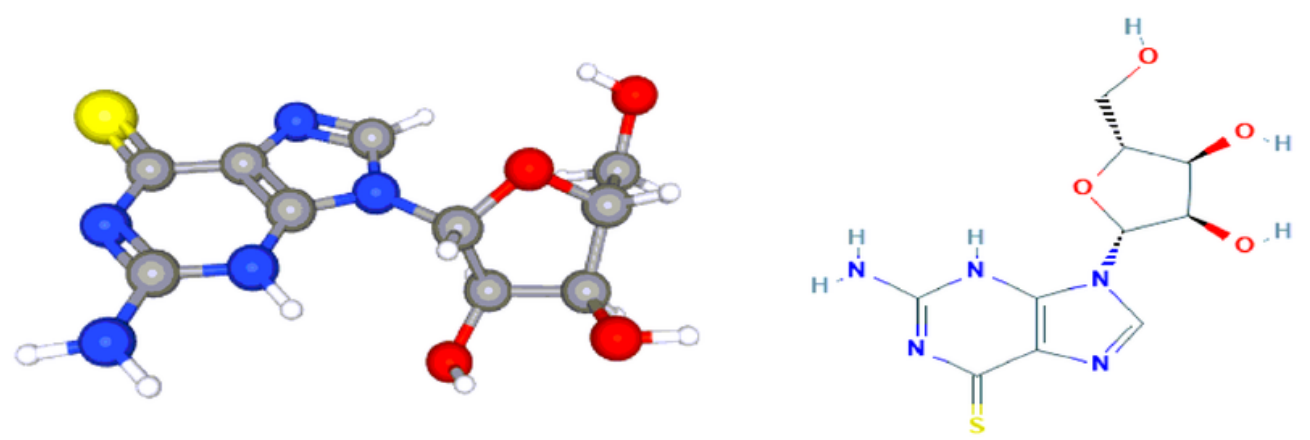

6-Thioguanosine $\mathrm{C}_{10} \mathrm{H}_{13} \mathrm{~N}_{5} \mathrm{O}_{4} \mathrm{~S}$ Compound CID: 2724387

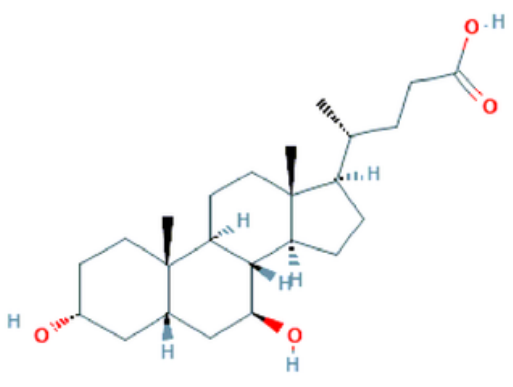

Ursodeoxycholic acid $\mathrm{C}_{24} \mathrm{H}_{40} \mathrm{O}_{4}$ Compound CID: 31401

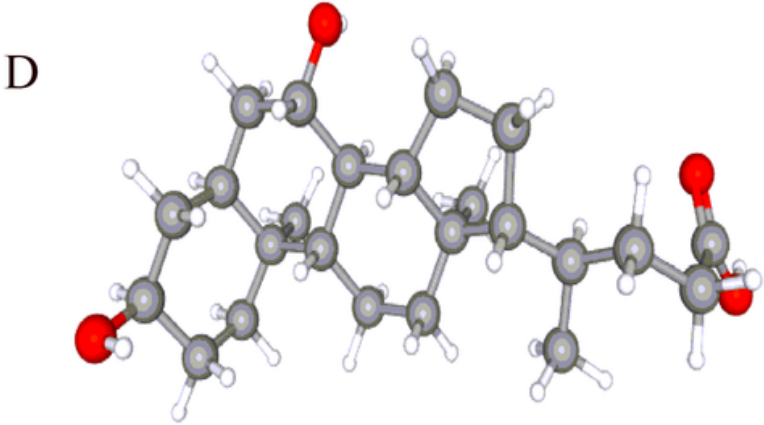

Figure 9

Small molecule compounds potentially inhibit TIMELESS expression. (A): gw8510; (B): 8-azaguanine; (C) 6-thioguanosine; (D): ursodeoxycholic acid.

\section{Supplementary Files}

This is a list of supplementary files associated with this preprint. Click to download.

- TableS1.docx 
- Tables2.docx

- Tables3.docx 